\title{
Models of resource planning during formation of calendar construction plans for erection of high-rise buildings
}

\author{
Irina Pocebneva ${ }^{1, *}$ Vadim Belousov, and Irina Fateeva ${ }^{1}$ \\ ${ }^{1}$ Voronezh State Technical University, Moscow Avenue, 14, Voronezh, 394026, Russia
}

\begin{abstract}
This article provides a methodical description of resource-time analysis for a wide range of requirements imposed for resource consumption processes in scheduling tasks during the construction of highrise buildings and facilities. The core of the proposed approach and is the resource models being determined. The generalized network models are the elements of those models, the amount of which can be too large to carry out the analysis of each element. Therefore, the problem is to approximate the original resource model by simpler time models, when their amount is not very large.
\end{abstract}

\section{Introduction}

Generalized network models (GNM) are a convenient tool that allows using mathematical methods efficiently in calendar planning and construction management when erecting high-rise buildings and structures. However, network methods do not allow a comparative analysis of the alternatives that arise when assigning resources that ensure the performance of these specific works. A mathematically correct approach to solving resource allocation problems is the combination of resource and time analysis in the process of plan formation. The problem of using the branch and bound method for the case, when the selection of workflows with indivisible resources is demanded, became the main topic of many works. However, the necessity to consider resource alternatives imposes the necessity to abandon the network setting of tasks. At the same time, a turning back to network models and their generalizations at a qualitatively new level allows the use of network methods [1]. This paper presents the resource-time analysis as a combination of resource analysis (i.e. the implementation of a sequence of RM transformations, including branching for a partial resource assignment) and time analysis (i.e., obtaining estimates for selecting the most promising branch node).

\section{Materials and Methods}

Assume that two finite sets $X, Z$ are given, where $X \neq t, X \cap Z \neq t$.

The space of functions of dimension $|X|+|Z|$ is denoted by $\Theta$. The functions are defined

\footnotetext{
* Corresponding author: ipocebneva@vgasu.vrn.ru
} 
on the set $X \bigcup Z$. The arbitrary function $\mathrm{T} \Theta$ will be called a plan.

Each $\mathrm{x}$ element of the $\mathrm{X}$ set is interpreted as an identifier of an event, which can imply the beginning, the end of the work or its parts, the construction stages, etc. For the T plan, the $T_{x}$ coordinate is the time for the event $x$ to occur. Each element $z \in Z$ is understood as a resource identifier with a continuous intensity scale. The $T_{z}$ number, which will be further called "tempo", is the reciprocal of the intensity. It is assumed that in the process of performing the work, the tempo of $\mathrm{T}_{\mathrm{z}}$ cannot be changed, although it can be selected from this range $\left[l_{z}, L_{z}\right]$ in the planning phase [2]. The duration of work $(x, y)$ is equal to $b_{z}$ where $\mathrm{b}$ is the volume of resource consumption $\mathrm{z}$ at work $(\mathrm{x}, \mathrm{y})$. If the resource usage rate $\mathrm{T}_{\mathrm{z}}$ is determined, the duration of the work that consumes this resource is automatically determined.

Sometimes it is convenient to understand the plan $\mathrm{T}$ as a vector of dimension $|\mathrm{X}|+|\mathrm{Z}|$ with the coordinates $\left\{\mathrm{T}_{\mathrm{x}}\right\}_{\mathrm{x}} \in \mathrm{x} \bigcup \mathrm{z}$.

Definition 1. The following system of inequalities is called a resource scale:

$$
\left\{l_{z} \leq T_{z} \leq L_{z}\right\}_{z \in Z}
$$

for the plans T, where $\left\{l_{z}\right\}_{z \in Z}$, and $\left\{L_{z}\right\}_{z \in Z}$, answer the correlations $0 \leq l_{z} \leq L_{z}$.

Definition 2. The temporary model (BM) $\mathrm{S}$ is given when the resource scale (1) is defined as well as the finite system of conditions of the following form:

$$
\begin{aligned}
T_{x} \leq L \quad(x \in X) \\
-T_{x} \leq-1 \quad(x \in X) \\
T_{x}-T_{y} \leq a \quad(x, y \in X, x \neq y), \\
T_{x}-T_{y} \leq b T_{z} \quad(x, y \in X, x \neq y, z \in Z),
\end{aligned}
$$

where on the right sides are arbitrary numbers $\mathrm{L}, 1, \mathrm{a}, \mathrm{b}$, with $\mathrm{b}>0$.

Definition 3. For a BM S, a plan $\mathrm{T}$ is said to be $\mathrm{S}$-admissible if answers all relations occurring in $\mathrm{S}$ of the form (1) - (5). The set of all S-admissible plans is denoted by $\mathrm{D}(\mathrm{S})$.

Using the $\mathrm{S}$ temporary model, a wide range of scheduling tasks can be formulated and solved. Note that all relations (1) - (5) are linear. This allows to use linear programming methods to solve problems with linear criteria $F=\sum_{x \in X \cup Z} \lambda_{x} T_{x}$ relatively to $\left\{T_{x}\right\}_{x \in X \cup Z}$.

The optimized criterion can be vector. The following problem is the example a vector minimization problem:

$$
\left\{T_{x}\right\}_{x \in X \cup Z} \rightarrow \min
$$

where the time limits of the events are minimized and the intensity of work is maximized. In certain cases, when solving a multicriteria problem, the plans obtained are not only answering the Pareto condition, but also not being improved by any of the criteria [3]. An example is the problem of finding early plans on a computer equivalent to a GNM in the following formulation:

$$
\left\{T_{x}\right\}_{x \in X} \rightarrow \min
$$

Definition 4. A resource model (RM) is a finite aggregate of TM:

$$
R=\left\{S_{\alpha}\right\}_{\alpha \in \sigma},
$$

where $\sigma^{*}$ is a finite set of resource alternatives.

Definition 5. $\mathrm{R}^{*}$ is the initial resource model, when

$$
R^{*}=\left\{S_{\alpha}^{*}\right\}_{\alpha \in \sigma^{*}},
$$

where $\sigma *$ is the set of variants with the full assignment of resources. 
Definition 6. For PM (6) we denote by

$$
D(R)=\bigcup_{\alpha \in \sigma}\left(S_{\alpha}\right)
$$

Plans $T \in D(R)$ will be called R-admissible.

A number of scheduling tasks can be set on the resource model. The example of an optimization task that is solved under the scheduling of work complexes is the following task: for a resource model (RM) R, R-admissible plan should be found that provides an optimum for some vector criterion $\mathrm{F}$.

Direct analysis of the set $\mathrm{D}(\mathrm{R})$ is often not possible because of the large number of alternatives for resources, and possibly for the intensity of their use.

\section{Results}

One of the approaches to solving such problems is connected with the application of the scheme of branches and boundaries [4]. For the effective use of it is necessary to specify the methods of branching ( dividing the set into subsets), the methods for obtaining estimates on subsets in accordance with $\mathrm{F}$ criterion. The way to divide RM into parts and approximate them by temporal and generalized network models for obtaining estimates by means of a sequence of corresponding transformations is shown below.

Definition 7. Assume that RM R' depends on the resource model $R\left(R^{\prime}<R\right)$ when $D\left(R^{\prime}\right)$ $\subset \mathrm{D}(\mathrm{R})$. When herewith $\mathrm{D}\left(\mathrm{R}^{\prime}\right) \neq \mathrm{D}(\mathrm{R})$, then the model $\mathrm{R}^{\prime}$ will be called more rigid than the model $R$. When $D(R)^{\prime}=D(R)$, then the models $R$ and $R^{\prime}$ are called equivalent .

The concept of subordination is obviously a partial order relation on the RM family (the properties of transitivity, reflexivity and antisymmetry are fulfilled).

The set $\mathrm{D}\left(\mathrm{R}^{*}\right)$, where $\mathrm{R}^{*}$ is the original model, is divided into a sufficiently large finite number of subsets $\mathrm{D}\left(\mathrm{S}_{\alpha^{*}}\right)$ satisfying the systems of linear inequalities. Using some properties of the model (for example, the partial assignment of resources), $\mathrm{R}^{*}$ can be decomposed into subsets for which $\mathrm{D}\left(\mathrm{R}_{1}\right), \ldots, \mathrm{D}\left(\mathrm{R}_{\mathrm{k}}\right)$ are such that $\mathrm{D}\left(\mathrm{R}^{*}\right)$ contains each of $\mathrm{D}\left(\mathrm{R}_{\mathrm{i}}\right)$ and each $\mathrm{D}\left(\mathrm{S}_{\alpha^{*}}\right)$ belongs to some $\mathrm{D}\left(\mathrm{R}_{\mathrm{i}}\right)$. Therefore, $\mathrm{R}_{\mathrm{i}}$ is a $\mathrm{RM}$ which is intermediate between the original RM and TM $S_{\alpha^{*}}$. The $R_{i}$ sets are constructed in such a way that the prospects of the plans included in $\mathrm{D}\left(\mathrm{R}_{\mathrm{i}}\right)$ can be estimated, for example, on the basis of the time analysis $R_{i}$ sufficiently accurate. When performing this analysis, $R_{i}$ is replaced by $S$ which is less rigid than or equivalent to TM. Such a replacement can be carried out gradually by elementary operations by constructing the sequence $\mathrm{R}^{\mathrm{i}}<\mathrm{R}^{\prime}<\mathrm{R}^{\prime}{ }^{\prime}<\ldots<$ S.

Note that each constraint (1) - (4) is determined by the linear form $\mathrm{f}(\mathrm{T})$ on the left side and the value on the right side. There are not more than 2 of such forms $(|\mathrm{X}|+|\mathrm{Z}|)+|\mathrm{X}|(|\mathrm{X}|$ $-1)$.

Suppose the linear form $\mathrm{f}(\mathrm{T})$ and $\mathrm{RM} R$ are given. Consider the variants of the occurrence of the form $\mathrm{f}(\mathrm{T})$ in $\mathrm{S}_{\alpha} \in \mathrm{R} . \mathrm{S}_{\alpha}$ can be contained in one or more inequalities of one of the forms (1)-(4), in which there is $\mathrm{f}(\mathrm{T})$. Of the two such inequalities $(\mathrm{T})<\mathrm{A}, \mathrm{f}(\mathrm{T})<\mathrm{A}^{\prime}$, that one for which the right side is larger can be discarded (when $A^{\prime} \geq A$, the second inequality is needless). In some cases (considering the further transformation of the RM), it is advisable to leave the second inequality in order to discard the first, moving to a less rigid RM. It is worth noting that the set of occurrences of the form $f(T)$ in $S_{\alpha}$ may turn out to be empty.

\section{Discussion}

There are various ways to change over to a simpler and yet equivalent RMs.

Thus, if for some $z \in Z$ we have $1_{z}=L_{z}$, then (5) can be replaced by two inequalities of the form (4): 


$$
\left\{\begin{array}{l}
T_{x}-T_{y} \leq b L_{z}, \\
T_{y}-T_{x} \leq-b l_{z}
\end{array}\right.
$$

which facilitates conducting temporary analysis.

Operation of a fictitious extension of the scope of use of the resource.

Suppose that the resources $z, z$ 'are used in BM S at work $(x, y)$. Then Tx-Ty $=b 1 T z$ and $\mathrm{Tx}-\mathrm{Ty}=\mathrm{b} 2 \mathrm{Tz}$. In addition, let $\mathrm{z}$ 'be used at work $\left(\mathrm{x}^{\prime}, \mathrm{y}^{\prime}\right)$ : $\mathrm{Tx} \mathrm{x}^{\prime}-\mathrm{Tu} \mathrm{\prime}^{\prime}=\mathrm{b} 3 \mathrm{Tz},$. We add the equality $\mathrm{Tx},-\mathrm{Ty},=\mathrm{b} 4 \mathrm{Tz}$, where $\mathrm{b} 4=\mathrm{b} 1 \mathrm{~b} 3 / \mathrm{b} 2$. We obtain a BM equivalent to $\mathrm{S}$.

The operation of matching the tempo range. Let the resources $\mathrm{z}, \mathrm{z}$ 'be used in the BM at work $(x, y)$.

It means that $l \leq T_{z} \leq L, l \leq T_{z^{*}} \leq L^{\prime}, T_{x}-T_{y}=b T_{z}, T_{x}-T_{y}=b^{\prime} T_{z^{\prime}} \cdots$

It is reasonable to consider the intersection of tempo ranges. Instead of the inequality $l \leq T_{z} \leq L$ while working with models, the following inequality should be taken:

$$
\max \left\{l, \frac{b^{\prime} l}{b}\right\} \leq T_{z} \leq \min \left\{L, \frac{b^{\prime} L}{b}\right\} .
$$

TM which is equivalent to the original one is obtained similarly for $T_{z^{\prime}}$.

Operation of fragmentation of the tempo range.

Suppose that for $\mathrm{S}_{\alpha} \in \mathrm{R}$ when some $\mathrm{z} \in \mathrm{Z}$, there is the strict inequality $1_{\mathrm{z}}<\mathrm{L}_{\mathrm{z}}$. Consider 1 $\in\left(l_{z} L_{z}\right)$ and replace $T M S_{\alpha}$ by two BMs $S_{\alpha}$ and $S_{\alpha}$ " that differ from $S_{\alpha}$ in that the inequality (1) is replaced by $1_{z}<T_{z}<1$ and in $S_{\alpha}$ " by $1<T_{z}<L_{z}$ under a condition of given $\mathrm{z}$ in $\mathrm{S}_{\alpha}$

The operation of fragmentation enables obtaining RM R', which is the equivalent to $R$ model, for using it later in the branch and boundary analysis. In the limiting case, a change of TM to an infinite set of GNM ${ }_{\mathrm{S} \alpha}$ ' with a fixed rate of resource consumption is obtained, i.e. with equalities $1_{z}=L_{z}$ and inequalities (7).

The following operations make it possible to obtain less rigid RMs.

Generalization operation.

Suppose that PM (6) and the form $f$ are given. The inequality $f(T) \leq A$ is assumed to be general for $\mathrm{R}$ if it occurs in all $\mathrm{S} \alpha$. Other inequalities containing fare assumed to be special. We define a new $R M R^{\prime}>R$ by discarding all the partial inequalities containing f from $R$. This procedure will be called the operation of generalization. If some $S_{\alpha}$ does not contain an inequality with $\mathrm{f}$ form, then $\mathrm{R}$ ' also does not contain any of such inequality. When each $\mathrm{S}_{\alpha}$ contains at least one such inequality, then it is useful to include the inequality $f(T) \leq M$ in each $S_{\alpha}$, where $M$ is the maximum of right sides in all occurrences of $f$ into $R$. In this case, all $S_{\alpha}$. will contain this inequality, wherein they will not contain any other inequalities of $f$ form.

The operation of merging the tempo range.

This operation is a special case of a generalization operation with preliminary maximization of the right sides. It concerns inequalities (1) for a fixed $z$. Each $S_{\alpha} \in R^{\prime}$ is obtained of $\mathrm{S}_{\alpha}$ by replacing inequality (1) by $1 \mathrm{z}^{*}<\mathrm{Tz}^{*}<\mathrm{Lz}^{*}$, where $l_{z^{*}}=\min _{\alpha} l_{z}$, $L_{z^{*}}=\max _{\alpha} L_{z}$.

Operation of transition to a variable rate.

In TM S, equalities (5) for fixed $\mathrm{T}_{\mathrm{z}}$ are replaced by two inequalities (8):

$$
\left\{\begin{array}{l}
T_{x}-T_{y} \leq b L_{z}, \\
T_{y}-T_{x} \leq-b l_{z}
\end{array}\right.
$$

If the $\mathrm{z}$ resource served only one work $(\mathrm{x}, \mathrm{y})$, the new TM $\mathrm{S}^{\prime}$ would be equivalent to $\mathrm{S}$, since the tempo can be arbitrary. However, if there are several works serviced by the resource $z$, then the inequalities (8) mean that the rate of use of the resource $z$ is set for each 
work. Thus, we pass to a less rigid TM. The usefulness may consist in that when we make a variable rate for every resource, there will not be any equality of type (5) in the TM, and it will turn into an GNM, which is more convenient in terms of time analysis.

Until now, we have considered the ways to reduce rigidity in order to obtain an "optimistic" assessment of the RM. The reasonability of further branching is also determined by the "pessimistic" assessment. If these two assessments are close, then there is not much sense in further branching indeed. A "pessimistic" assessment can be obtained by moving to a more rigid RM and then by the sequent time analysis of this RM. One of the methods for obtaining a more rigid RM is the resource assignment operation.

Assignment operation of $\mathrm{z}$ resource.

We take an arbitrary value from the rate $T_{z}$ in the TM S within the limits defined by inequality (1), and replace (1) by $\mathrm{c} \leq \mathrm{T}_{\mathrm{z}} \leq \mathrm{c}$. Now inequalities (3) can be replaced by a pair of inequalities $\mathrm{T}_{\mathrm{z}} \leq \mathrm{c}$ and $-\mathrm{T}_{\mathrm{z}} \leq-\mathrm{c}$.

Assigning all the resources $z \in Z$, we obtain the GNM, for which it is easy to conduct a time analysis and, if feasible plans exist, get a "pessimistic" estimate.

Since each $\mathrm{S}_{\alpha} \in \mathrm{R}$, which is considered as $\mathrm{RM}$, is more rigid than $\mathrm{R}$, the rigidifying of resources in $\mathrm{S}_{\alpha}$ will result in obtaining the GNM which is also more rigid than $\mathrm{R}$. This is sufficient for a pessimistic assessment of $\mathrm{R}$.

Consider the peculiarities that arise when different types of resource constraints are taken into account both at the initial stage of determining the initial RM and at the stages of its partitioning into TM subsets.

Assume that resource $\mathrm{z}$ is used at several works (or their parts)

$$
\left(x_{1}, y_{1}\right), \quad\left(x_{2}, y_{2}\right), \ldots, \quad\left(x_{n}, y_{n}\right)
$$

and can have any intensity from the segment $\left[\mathrm{I}_{0}, \mathrm{I}_{1}\right]$ of the positive semi axis. This means that the resource can be consumed at any rate from the interval $[1, \mathrm{~L}]$, where $1=$ $1 \backslash \mathrm{I}_{1}, \mathrm{~L}=1 \backslash_{0}$.

However, if the tempo is chosen, then it is considered unchanged within the process of executing the serviced $\mathrm{z}$ resources of works.

Assume that $b_{1}, b_{2}, \ldots, b_{n}$ are the volumes of resource consumption in works (9). Using the resource at the tempo of $T_{z} \in[1, L]$ leads to the fact that $b_{1} T_{z}, b_{2} T_{z}, \ldots, b_{n} T_{z}$, of time units will be expended on works respectively (9), which is described by inequalities (1) and (5).

In fact, there are no alternatives, if we are satisfied with TM (1)-(5). They will occur only if in the time analysis there will arise a need to get rid of non-networked equalities (5).

With the operation of moving to a variable rate, one can get a less rigid GNM and analyze it. If this estimate is preliminary, the fragmentation of the tempo range should be made by dividing the range $[1, L]$ into two segments, for example $\left[l, \frac{L+l}{2}\right]$ and $\left[\frac{L+l}{2}, L\right]$. The time analysis of each new more rigid TM will undoubtedly be more accurate. The division can be continued until the boundaries [1', L] become almost indistinguishable.

The case of a discrete set of intensities. Suppose that instead of the range [1, L], a finite set of permissible usage rates of the resource $\Lambda=\left\{\lambda_{1}, \ldots, \lambda_{m}\right\}, \quad 0<\lambda_{1}<\ldots<\lambda_{m}$ is and any $T_{z} \in \Lambda$ can be chosen. After such a choice, there will be equality of a network character:

$$
\mathrm{T}_{\mathrm{xi}}-\mathrm{T}_{\mathrm{yi}}=\mathrm{b}_{\mathrm{i}} \mathrm{T}_{\mathrm{z}} \text {. }
$$

As a result, $\mathrm{m}$ GNM is obtained for each $\mathrm{z}$. The $\mathrm{m}$ number can be found to be too large. In this case, the TM with the inequality (1) of the following form can be investigated similarly to the way it was done in the previous section:

$$
\lambda_{1} \leq T_{z} \leq \lambda_{m}
$$

By allowing $\mathrm{T}_{\mathrm{z}}$ to take continuous values (10) instead of discrete values, we get a linear 
problem instead of a search problem. In order to obtain a more accurate estimate, the fragmentation operation can be replaced by considering more rigid TMs corresponding to the two tempo intervals $\left[\lambda_{1}, \lambda_{\mathrm{i}}\right]$ and $\left[\lambda_{\mathrm{i}+1}, \lambda_{\mathrm{m}}\right]$.

Suppose that there is an indivisible $\mathrm{z}$ resource that serves a number of works or their parts $\left(\mathrm{x}_{1}, \mathrm{y}_{1}\right), \ldots,\left(\mathrm{x}_{\mathrm{n}}, \mathrm{y}_{\mathrm{n}}\right)$, wherein the order of their execution is not defined in advance. Suppose $\pi$ is a permutation of numbers $1, \ldots, \mathrm{n}$ from $\mathrm{n}$. In order to require the execution of work in the order

$$
\left(x_{\pi_{1}}, y_{\pi_{1}}\right), \ldots, \quad\left(x_{\pi_{n}}, y_{\pi_{n}}\right),
$$

it is enough to set the constraints of transport type:

$$
T_{y_{\pi_{1}}} \leq T_{x_{\pi_{2}}}, T_{y_{\pi_{2}}} \leq T_{x_{\pi_{3}}}, \ldots, T_{y_{\pi_{n-1}}} \leq T_{x_{\pi_{n}}}
$$

The number of interchanges grows rapidly with increasing $n$. Therefore, there is a need for a gradual fragmentation of the set of interchanges during the investigation of the RM for a given $\mathrm{n}$. This can be done indirectly by the sequent including of inequalities (12).

At the first step suppose dividing $\mathrm{R}=\left\{\mathrm{S}_{\alpha}\right\}$ into two classes $\mathrm{R}^{\prime}$ and $\mathrm{R} "$. We assign all the $\mathrm{S}_{\alpha}$ for which $\mathrm{T}_{\mathrm{y} 1}<\mathrm{T}_{\mathrm{x} 2}$, to $\mathrm{R}$ ', to the second class we assign all the $\mathrm{S}_{\alpha}$ for which $\mathrm{T}_{\mathrm{y} 2}<\mathrm{T}_{\mathrm{x} 1}$,

Continuing the branching in the $\mathrm{R}^{\prime}$ class, we add one of the inequalities $\mathrm{T}_{\mathrm{y} 1}<\mathrm{T}_{\mathrm{x} 3}$, or $\mathrm{T}_{\mathrm{y} 3} \leq \mathrm{T}_{\mathrm{x} 2}$, to $\mathrm{T}_{\mathrm{y} 1}<\mathrm{T}_{\mathrm{x} 2}$. If $\mathrm{T}_{\mathrm{y} 1}<\mathrm{T}_{\mathrm{x} 3}$ is chosen, in the next step we consider the inequalities $\mathrm{T}_{\mathrm{y} 2}$ $<\mathrm{T}_{\mathrm{x} 3}$ or $\mathrm{T}_{\mathrm{y} 3}<\mathrm{T}_{\mathrm{x} 2}$. After choosing the second of these inequalities, the inequality $\mathrm{T}_{\mathrm{y} 1}<\mathrm{T}_{\mathrm{x} 2}$ becomes needless, so it can be discarded. Obviously, a binary branch can be used to view the entire set of interchanges.

In cases when the downtime of a resource is inadmissible, along with the definition of the order of servicing of works (11), it is worth pointing out that the next work should begin immediately after the end of the previous work. Formally, this means that inequalities (12) should be supplemented by the "opposite" inequalities:

$$
T_{x_{\pi_{2}}} \leq T_{y_{\pi_{1}}}, T_{x_{\pi_{3}}} \leq T_{y_{\pi_{2}}}, \ldots, T_{x_{\pi_{n}}} \leq T_{y_{\pi_{n-1}}}
$$

Here, with the gradual assignment of inequalities (12), (13), a more subtle approach is required. Suppose, for example, that $\mathrm{T}_{\mathrm{y} 1} \leq \mathrm{T}_{\mathrm{x} 2}$ is chosen at the first step. Then, choosing $\mathrm{T}_{\mathrm{x} 2} \leq \mathrm{T}_{\mathrm{y} 1}$ at the second step, we clearly indicate that the second work should be performed immediately after the end of the first work. An alternative to this does not have a simple expression by the formulas (4), that is why one has to use a strict inequality, meaning to insert obligatorily some other work between the first and second work in the future.

One of the approaches consists in firstly fixing the work that will be performed first, then the work that will be the second, etc. The most effective way is determined by the specific features of the problem. For the task of determining the order of work at construction sites with continuous work of the performers, a detailed algorithm and some results of computer calculations are described in $[5,6]$. It should be noted that several resources can be used on the same work. There are no obstacles to the theoretical apparatus proposed here. Moreover, the same resource can belong to two types $(\mathrm{P}, \mathrm{Q}),(\mathrm{P}=3.1,3.2$, $\mathrm{Q}=3.3,3.4$ - section numbers). This means that there is a need to determine both the rate of resource consumption, and the order of maintenance work. Such a resource can be treated as two different resources. When using the scheme of branches and boundaries, instead of full detailing, first one resource, then another, etc., in a number of problems, it is advisable to alternate a partial assignment to different resources [7].

\section{Conclusion}

The paper considers ways of transforming the scheduling tasks: decomposition, aggregation, etc. The modern theory of structured programming and the principles of developing software complexes largely use the ideas of Descartes. The same ideas comprise 
the core of the method of branches and boundaries. This paper considers methods of transformation for approximating the scheduling tasks by linear and network tasks. The present work shows that the branching (decomposition) within the formation of calendar schedules for erecting high-rise buildings and structures should be considered in close conjunction with aggregation which is the reverse operation. Resource-and-time analysis is presented as a flexible process, involving a dialogue, in one respect, with consumers (specialists in the field of organization, technology and production management of unique works with the aim of adjusting resource and network constraints), and, in other respect, with a computer while conducting resource and time analysis of the RM, its parts or their transformations.

\section{References}

1. V.V. Gluhov, I.V. Ilin, Lecture Notes in Computer Science, 8638, 509- 518 (2014) DOi-10.1007/978-3-319-10353-2_46

2. I.V. Ilin, A.I. Levina, O.Yu. Iliashenko, MATEC Web of Conf, 86, 05028 (2016) DOi $10.1051 /$ matecconf $/ 20168605028$

3. S.A. Barkalov, Nguen Van ZHang, Nguen Than' Zhang, Sistemy upravleniya i informacionnye tekhnologii, 3.1(53), 116-119 (2013)

4. V.E. Belousov, Ekonomika i menedzhment sistem upravleniya, 3.2(17), 227-232 (2015)

5. V.E. Belousov, Sistemy upravleniya i informacionnye tekhnologii, 4(62), 31-33 (2015)

6. R. Bril, O.V. Kalinina, I.V. Ilin, A.S. Dubgorn, O.Yu. Iliashenko, SCM, 692- 693 (2017) DOi - 10.1109/SCM.2017.7970692

7. E. Nezhnikova, Procedia Engineering, 165, 1300-1304 (2016) doi 10.1016/j.proeng.2016.11.854 